\title{
Information Technology in the Interventional Pain Practice: Electronic Medical Records, Practice Management Software, AND DoCument Management
}

\begin{abstract}
$\frac{\text { Standiford Helm, MD, and Hans C. Ha }}{\text { Electronic medical records (EMR) can }}$ both replace the paper clinical chart and perform scheduling and billing tasks. EMRs are currently used in only a small proportion of medical practices; however, EMR adoption is expected to soar in a few years. Governmental and industry concerns about safety and quality, along with medical practice needs for increased productivity, are fueling this transition.

Medical practices are likely to consider transitioning to EMRs. At this point, the marketplace is fragmented in terms of suppliers, and refinement of the software is continu-
\end{abstract}

ing. Transitioning to an EMR is a step that offers enormous benefits to interventional pain management physicians, in terms of compliance, the ability to maintain quality, and the ability to manage practices, including larger and more complex practices. Transitioning to an EMR is also a major effort in terms of time and expense: The goal is to settle on a specific EMR and continue to use it.

This review focuses on (1) why physicians are or are not transitioning to EMRs; (2) the benefits of a transition; and (3) factors to consider in evaluating the various competing software products. Although EMR technology continues to develop, the review also considers areas in which future development is necessary. The most important areas are seen in document management, data input, and outcomes analysis and decision support capability.

The EMR market is evolving rapidly. However, this review should in no way serve as an endorsement of any particular system, vendor, or technology.

Keywords: Interventional pain practice, information technology, electronic medical records
The management of medical practices is undergoing an enormous change, with a transition from paper to electronic medical records occurring as the benefits of information technology are being introduced into physicians' offices. As in other sectors of the economy, practices that do transition to an EMR will face an advantage in the marketplace $(1,2)$.

The transition from a paper to a digital record is a complex process, requiring care and study to assist in choosing the system, which provides the necessary features and support at an appropriate cost. A good understanding of EMR technology is useful. It not only allows the practice to retain the chosen system and avoid the aggravation and expense of implementing a second system but offers advantages in compatibility and the ability to cross-talk

From Pacific Coast Pain Management Center, Lake Forest, California, and The Pain Relief Centers, PA, Conover, North Carolina. Address Correspondence: Standiford Helm II, MD, Pacific Coast Pain Management Center, 23792 Rockfield Blvd, Suite 101, Lake Forest, CA 92630.

E-mail: drhelm@pcpmc.com

Funding: No outside funding was provided in preparation of this manuscript.

Conflicts: Dr. Helm is a shareholder in ChartLogic, Inc., an EMR software developer.

Acknowledgement: Manuscript received on 3/2/04. Revision submitted on $3 / 13 / 04$. Accepted for publication on $3 / 20 / 04$. with systems in hospitals and pharmacies and with payors (3).

Physicians have historically been perceived as high-level practitioners who exploit technology and use each new advance as an application to enhance care. In fact, physicians lag behind most other professionals in the use of information technology. For example, patients can submit questions to a physician by E-mail in only $10-12 \%$ of primary-care practices, and data analysis to determine future course of care is not even a consideration in most clinicians' plans for future development (4).

EMRs are currently used in fewer than $10 \%$ of medical practices; however, EMR adoption is expected to soar to $50 \%$ in fewer than five years, with the bulk of this increase coming from practices of eight or fewer physicians (5). Figure 1 shows the anticipated growth in spending by practice size (6).

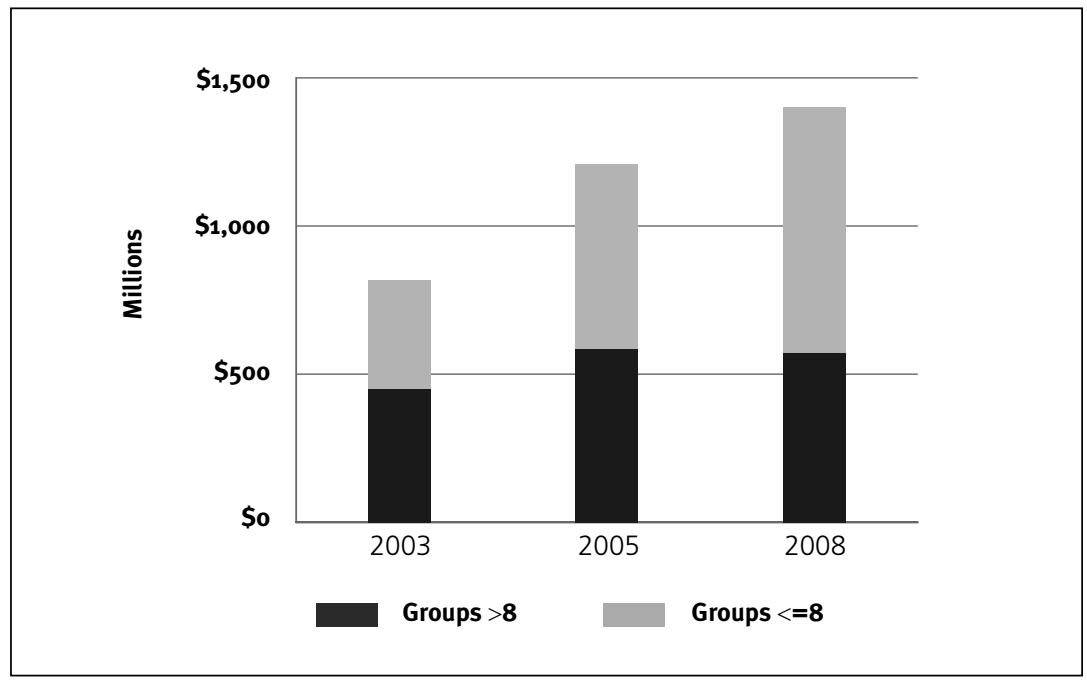

Fig 1. Growth in spending for EMRs by practice size 


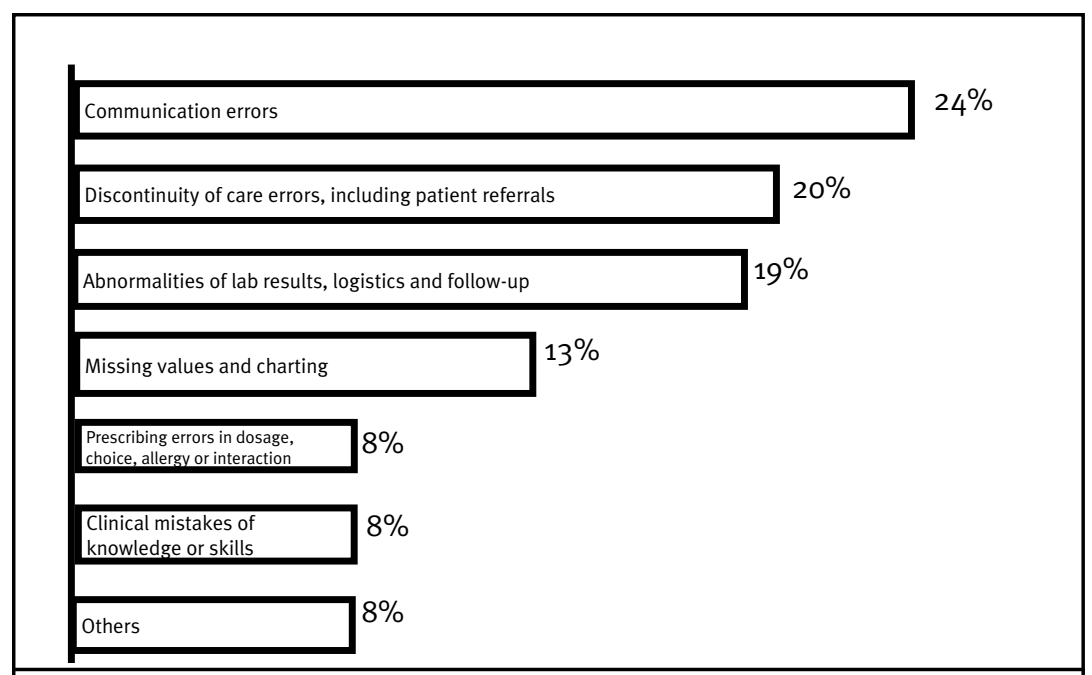

Fig 2. A Breakdown of errors in outpatient settings

\section{WHY EMR Now?}

The move toward EMRs and the use of information technology in medical practices are being driven by multiple factors. Medical errors are recognized to be a major cause of unnecessary hospitalizations and increased medical costs. The Institute of Medicine has estimated that more than 7,000 deaths and up to $7 \%$ of hospital admissions occur as a result of adverse drug effects and that up to $95 \%$ of these adverse drug events could be avoided using computerized data entry (7). Figure 2 presents the types and prevalence of errors in a family-practice setting (3). These errors occur primarily from loss of information or lack of follow-up. Computerization of the medical office is ideally situated to minimize errors of this type.

Table 1. IOM-Proposed Core Functions of EMRs

Health information and data: Patients' diagnoses, allergies, and laboratory test results

Results management: New and past test results by all clinicians involved in treating a patient

Order management: Medications, tests, and other services

Decision support: Electronic alerts and reminders to improve compliance with best practices, ensure preventive practices, identify possible drug interactions, and facilitate diagnoses and treatments

Electronic communication and connectivity: Secure and readily accessible communication among clinicians and patients

Patient support: Tools offering patients access to their medical records, interactive education, and the ability to do home monitoring and self-testing

Administrative processes: Tools, including scheduling systems, that improve administrative efficiencies and patient service

Reporting: Electronic data storage that uses uniform data standards to enable physician offices and health care organizations to comply with federal, state, and private reporting requirements in a timely manner
(HIPAA) created standards for insurance and billing (11). This effort is now moving to internal medical office functioning, with the Institute of Medicine having delineated the components that an EMR should fulfill. These components are shown in Table 1. They have been sent to HL7 to create computer standards for these tasks so that software developers will have an open platform for future development (12).

Internally, medical practices are under increasing financial and regulatory pressure. The possibility of revenue streams increasing at a rate less than that of inflation forces practices to look for increased productivity. Physicians have limited means of responding to financial pressures:

- Decrease overhead

- Enhance productivity, seeing more patients per day

- Add new revenue streams, such as facility fees, testing, and therapy

EMRs have the potential-but not the certainty-of assisting with all three goals.

EMRs should allow the practice to function both with fewer filing clerks and with fewer or no transcription costs (13). In addition, the direct costs of purchasing paper charts can be decreased or eliminated as a practice moves to a paperless environment. These savings are the greatest direct financial benefits of transitioning to any EMR.

Productivity may be enhanced. EMRs have the potential of shifting down a significant portion of chart preparation to lower-cost personnel, allowing physicians to focus on direct patient encounter. Space previously used by medical record storage can be shifted over to revenue-generating activities.

At the same time, physicians are under scrutiny for documentation and compliance, with the need to show that the level of service billed for was actually provided. The American Society of Interventional Pain Physicians (ASIPP) has comprehensively detailed what needs to be included in a report to meet billing requirements (14). The issue of compliance is compounded for interventional pain management physicians who issue controlled substances, as they must document that these prescriptions serve a valid medical purpose. 
Table 2. Functions Performed by Practice Management Software

\begin{tabular}{|l|}
\hline $\begin{array}{l}\text { All aspects of management of patient information, including demographic data, insurance } \\
\text { information, and the tracking of changes in demographics and insurance }\end{array}$ \\
\hline Appointment and procedure scheduling and rescheduling \\
\hline Communication with the EMR to capture charges and create billing forms \\
\hline $\begin{array}{l}\text { Creation of bills, either paper or electronic. Note that many insurers require paper bills } \\
\text { from interventional practices because of the requirement for copies of procedure notes. } \\
\text { Currently, no protocol exists for the electronic transmission of chart documents. }\end{array}$ \\
\hline Management of accounts receivable and collections \\
\hline Practice management reports
\end{tabular}

A growing concern is "Incident to" billing for physician extenders. Incident to refers to CMS guidelines detailed in statute $\$ 2050$, under which the services of midlevel practitioners, such as physician's assistants (PA) and nurse practitioners (NP), can be billed (15). Essentially, depending on the level of availability of the supervising physician, the PA's or NP's work can be billed at either $100 \%$ or $85 \%$ of the physician's fee. EMRs should allow a PA or NP to code the level of physician availability at the time the service was provided, to ensure compliance with CMS guidelines and to avoid OIG investigations.

Practices that have access to data have many advantages over their peers lacking such access. Digitalized offices have the potential to document outcomes easily. In large, interventional pain practices have not been documenting outcomes well, so anyone with these data will have a significant competitive advantage (16-18).

Quality can be maintained, with information easily and quickly shared between multiple sites and multiple providers. Solo physicians performing procedures away from their office will have ready access to an entire chart, including the rationale as to why a particular procedure is being performed, so that they are not dependent on the accuracy of scheduling clerks in determining what procedure is to be performed. Multipractitioner practices will be able to follow the treatment course of all patients easily, regardless of who has seen them.

Quality is also maintained by the reminder of appropriate health maintenance or screening procedures (19). In a pain management practice, these might be liver function tests, urine drug screens, or follow-up assessments of psychological status and function. On a more advanced level, treatment algorithms can provide suggestions of appropriate, evidencebased care. This benefit is useful for intro- ducing physicians new to the practice to the manner in which a particular practice evaluates the evidence and as a reminder to busy clinicians as to ideas that they may have neglected owing to the pressures of a busy day.

Quality of life is another major reason why physicians would want to transition to an EMR. Entering data is an issue for both paper and electronic charts. For many practitioners, this means dictating nights and weekends. The EMR should ideally allow the note to be finished close to the end of the patient encounter, freeing up the physician's time.

Thus, the introduction of information technology into medical practices is seen by government and industry as an answer to problems of quality and cost and by physicians as an answer to problems of quality, documentation, compliance, practice productivity, competitiveness, and quality of life.

\section{Workplace Tasks Performed BY THE EMR}

Workflow in the office is divided into front- and back-office activities. The front office deals with patient registration, scheduling, and billing. The back office deals with all aspects of the clinical encounter, including documentation of office visits, medication management, communications logs and information from other providers, and procedures.

Because the tasks carried out are similar to the functions necessary to run most businesses, the front office's activity was the first aspect of the medical record to be computerized, using what is called practice management software (PMS). Table 2 presents a detailed list of tasks carried out by PMS.

The current concerns regarding, and growth in the application of, information technology apply primarily to back-office functions. Table 3 presents a detailed list of tasks carried out by the EMR. This review uses the term $E M R$ interchangeably with electronic medical records, either coupled with or separate from PMS software, referring specifically to PMS as indicated.

\section{Why Not an EMR?}

Transitioning to an EMR can easily cost $\$ 50,000$ for software and hardware. A solo general practitioner who uses no transcription and has a small office staff can conclude that no revenue stream exists to divert for the transition. The interventional pain practitioner, because of the demands created by compliance and documentation, should at a minimum be using transcription. An EMR is a capital investment, with the costs spread out over at least the three-year cycle that computer hardware is expected to last. With this formulation, the EMR would cost some $\$ 1,500$ per month, a figure that can be far less than the monthly costs of transcription or of a filing clerk. The EMR therefore should be viewed like a fluoroscopy unit-a necessary capital expense of the practice (2).

The cost of an EMR cannot be evaluated without looking at potential tax benefits. Under the Internal Revenue Tax Code Section 179, a physician may now depreciate up to $\$ 100,000$ (up from $\$ 25,000)$ of equipment, including EMR computer hardware and software, in the year of purchase instead of over several years (20).

Table 3. Tasks Performed by EMRs
\begin{tabular}{|l|}
\hline Chart documentation \\
\hline Note transcription \\
\hline Prescription writer and database \\
\hline Order entry and results reporting \\
\hline Inpatient reports \\
\hline Telephone triage documentation \\
\hline $\begin{array}{l}\text { Secure messaging systems-electronic } \\
\text { mail }\end{array}$ \\
\hline $\begin{array}{l}\text { Ability to interface with practice } \\
\text { management software and ancillary } \\
\text { information systems }\end{array}$ \\
\hline $\begin{array}{l}\text { Decision support tools, including } \\
\text { evidence-based guidelines }\end{array}$ \\
\hline Patient registration information \\
\hline Support for multiple users \\
\hline Support for multiple databases \\
\hline Reporting capabilities \\
\hline Remote access to data \\
\hline
\end{tabular}


Practices may delay investing in EMRs because of imperfections in the technology: EMRs are a developing field. For example, data input with voice technology is a useful approach but not one that ensures absolutely accurate reports without proofreading, which does require operator acceptance $(21,22)$. Implementing an EMR will require a significant cost in physician learning time and in training of staff. As this cost has to be met, the appropriate time is probably now. A modular approach in introduction, beginning with a messaging system or electronic prescriptions, allows a physician's practice to step in slowly while waiting for further refinements in the software (23).

Not all patients will accept EMRs, particularly given the diversity of the American population in terms of age, ethnicity, and education, if they participate in entering data. Patients may require support in inputting data into computers, just as they require support in completing paper forms (24).

\section{Factors to Consider in Transitioning}

Evaluating the various EMRs is a difficult, complex process. Hundreds of EMR software developers exist, in addition to custom efforts made for specific pain practices. Although central clearinghouses exist, a multitude of data are available, and those data are in such flux that using the clearinghouses can be difficult (25). This creates a situation in which EMRs are sold rather than bought: Whichever vendor can bring a product to the practice's attention has a better chance of selling the product. Because of the importance of the transition to EMRs and the difficulty and expense of changing if the product purchased is not retained, an important factor for pain management physicians is to have an understanding of how to evaluate different software claims. This issue is particularly salient when one considers that anywhere from 50 to $95 \%$ of all practices transitioning to an EMR retain the first EMR they chose: The costs of choosing the $50 \%$ software are too high.

Among the issues to evaluate:

- Whether to get freestanding or combined EMR and PMS programs

- How the software handles workflow

- Data input, including accuracy

- Hardware and database needs

- How templates are developed

- Whether to buy from a developer or VAR
- The strength of the support program

- How multiple sites are handled

- Document management

- Practical considerations for the practice

The final point in evaluating software is to speak with a practice that is similar to yours and has already implemented the program.

\section{EMR and PMS: One Program or Two?}

Medical office work can be divided into front- and back-office functions, with PMS software handling the front-office and EMRs handling the back-office tasks. One can get either one program for each function or separate programs. The choice of the way to go termed the "best of breed versus integrated debate." Currently, no right answer exists. The best-of-breed camp argues that you can choose the software that best suits your needs for each purpose. By choosing the best-of-breed programs, physicians can avoid compromising one program at the expense of the other. Best-of-breed proponents suggest that integrated software favors either the PMS or EMR components.

One can find “integrated" programs, which are merely two separate programs with separate databases being sold under one cover. Those who favor the integrated program argue that they provide "all-inone" shopping. If any problem arises, physicians have to go to only one place for a resolution. Finger pointing by vendorsblaming each other for failure to communicate billing information between the EMR and the PMS software-also can be avoided.

\section{Assessing How Workflow Is Handled}

EMRs provide a technological means of fulfilling tasks that must be performed to run a medical office. Medical offices vary widely in the details of how they operate. If a practice is to retain the software, the software must provide easy means for both physicians and staff to complete their tasks.

In an initial comparison of programs, they all appear to do all things. One way of differentiating between programs is to ask how things are done rather than what is done. Some software is programmed to complete tasks on its own; other versions require the user to leave the EMR, retrieve a stand-alone program, and then complete the task. For example, consider the tasks of faxing and managing scanned documents. These responsibilities can be handled in many ways. Software which has these two functions as parts of the software foundation will be faster than an EMR which exports these functions to freestanding programs, such as WinFax or Adobe. An additional timesaver is that some software allows documents to be faxed directly to your computer. This means that the staff is working with digitalized files from the beginning rather than spending time scanning faxed documents or creating paper charts.

A related issue is whether the information required to complete a task is presented by the software. For example, if a request comes in from a pharmacy for a refill, how much effort is required to locate the patient's diagnosis, prescription history, and allergies? Another related issue is the ability to manage prescriptions and to interface with pharmacies. One major step to improving safety and minimizing drug errors is to transmit all prescriptions electronically. State board of pharmacy representatives have the elimination of paper prescriptions as their goal (26).

EMRs should have the capability to manage prescriptions and to interface efficiently with pharmacies. The major limitation on this ability is the DEA's requirement for a "live" signature for Schedule II medications. The DEA is working on adopting a protocol for digital certification of signatures; this would allow faxing of Schedule II prescriptions directly from the computer. This protocol will probably not be available until at least the middle of 2005 (15).

Management of scanned documents is a major workflow issue. It should be simple for staff to identify and quickly place scanned outside documents into the proper place in the file. Identifying and accessing new outside documents should be simple for the providers.

Using a program designed around workflow will be easier, and it is more likely to be retained than would a competitor, which pays less attention to the needs of the staff.

\section{Input}

All medical records-paper or digital-require that data be entered into them. Data input is a major expense in terms of time and money. Handwritten charts are a potential source of error and 
lost data. Transcription is time-consuming, often taking up a practitioner's weekends and evenings, and it is expensive.

EMRs need a data entry system that is fast and accurate. To date, no perfect solution exists. The current options include:

- Typing

- Voice recognition

- Dictation to a transcription service, with the transcribed notes either transmitted electronically into the record or scanned in by office staff

- "Drop-and-click" menus, with appropriate words or phrases being chosen from a drop-down list

These actions can be performed by a physician or by a scribe accompanying a physician. Some data can be entered before the visit, using pre-encounter forms filled out by a patient or medical records forwarded from another office. Patients can fill out paper forms with input by the staff or, with such technologies as Tablet PCs, enter the data directly into the software.

Voice recognition offers the greatest flexibility, but this method is hampered by lingering inaccurate recognition, the editing of which can be difficult. All words presented by voice recognition are spelled correctly, so that spell checking is ineffective. Efforts to proofread "on the run" are not entirely successful. Proofreading after the fact is expensive and diminishes the advantages of the EMR. However, voice recognition does have inherent benefits. Performed during the office visit, voice recognition offers the richest, most detailed and specific dictation because the notes are being recorded as a patient speaks. It does not create a lag during which details can be forgotten or during which all the patients of the day blend into one. Errors, such as the common mistake of substitution of left for right, can be corrected by patients as they listen to the dictation. Patients can also hear the discussion and understand the thought process by which a physician comes to conclusions. Generally, strong physician willingness to work with voice recognition is necessary for use of this technology. In time, voice recognition will become the preferred method of data entry as technology improves.

Although most EMRs claim the ability to work with voice recognition, a preferable approach is to work with an EMR that has this technology integrated at the programming level. Such EMRs are available.

Typing is a mainstay and often used in conjunction with EMRs. We have found, depending on how information is presented by individual patients, that sometimes typing is better than dictating data. Typing has the advantage of allowing the use of a spell-checking program. It also offers the advantages of contemporaneous transcription. For lengthy discussions, however, it is slow and tedious.

Drop-down lists are another option used by many programs. These lists may be adequate for common family practice complaints, but they fall short for both the history of present illness and discussion sections of a typical pain management consultation. The use of drop-down lists could lower retention rates of EMR systems for pain management practices. Specific programs reveal a superb understanding of office and physician workflow, but these same programs suffer because of an overreliance on drop-down lists.

A significant advantage of dropdown lists is that they offer an easy way of tracking data, as one can query the specific field in the database. In this way, difficult tasks, such as tracking outcomes, can be performed.

Physicians are comfortable with transcription, the current standard method for recording data. At the same time, the disadvantages of transcription remain; pitfalls include loss of timeliness by dictating after the fact and the ongoing expense of the method. A transcription level that allows the EMR to be implemented and retained may be a necessary requirement in certain practices. These practices are best served by an EMR which allows a transcriptionist to fax the transcribed note already in electronic format directly into the EMR. Then a staff member can file the note with just a few clicks of a mouse.

Given the lack of an ideal solution, EMRs should offer flexibility, so that each practice and practitioner can find the solution that best suits their needs.

\section{Hardware and Database Requirements}

Hardware issues, in the previously noncomputerized office, are a major concern. Although hardware costs are decreasing, the cost of networking an office is considerable. In addition, networks require maintenance, and the practice will need access to an information technolo- gy specialist to assist with the inevitable problems which will occur. The cost of the information technologist must be factored in when assessing the cost of a system. Our preference is to use workstations rather than standard PCs.

Given the extent to which you depend on your hardware and the amount of use it will be given, do not look at the hardware as a spot to save money. Buy from a reputable manufacturer with a good reputation for small business support. Anticipate that your hardware will have a three-year life and that, at the end of that time, new options will be available to enhance productivity in your office.

The stability and backup of your database is crucial. Anticipate that you will need a mirrored RAID (redundant array of inexpensive discs) storage device to handle the volume of data associated with a paperless, or almost paperless, office.

To create a fully functioning EMR capable of handling growth and allowing access by multiple users at multiple sites, your software should be built on a structured query language (SQL) database (27). SQL databases are the industry standard for large data storage and retrieval and are "enterprise level." Different echelons of security are built in to allow access by users with a HIPAA-friendly "need-to-know" approach. Remote offices capitalize on this sequel technology to transfer through a secure tunnel and synchronize with each site $(22,28)$. Alternative systems, such as Linux, are available but lack the same installed base or industry support.

The other alternative commonly used in low-end or "homemade" EMRs are alphanumeric programs, such as Word files. They can create legible, typewritten notes effectively. They are described as "note takers." Data cannot be extracted for decision support or outcomes management, and some simple tasks, such as maintaining an accurate medical history-important both for quality of care and for compliance with CMS billing requirements-may become difficult.

The options considered have all been based on the assumption that the data will be stored in the physician's office. An alternative, potentially attractive to cashstrapped practices concerned about initial capital outlays, is the application service provider (ASP). ASPs offer remote maintenance of the data and software accessed over the Web in exchange for a monthly 
fee. An advantage is that upgrades will be accomplished transparently to the medical office. This model has been suggested for wide use, including software as ubiquitous as word processing and spreadsheets. Prior to the model's gaining acceptance, common platforms are needed to allow users to migrate from one ASP to another. Currently, this common platform does not exist. In addition, the practice is subject to loss of data control (21). Further, the cost savings is factious, with monthly costs exceeding the initial cost amortized over a number of years (3).

\section{Templates}

The medical encounter follows a set structure, starting with chief complaint and ending with a treatment plan. This sort of repeated activity, whether for a new visit or a progress note, lends itself to templating. In like manner, procedure notes also lend themselves to templating. Templates allow the creation of a prestructured note. In programs that do not automatically calculate the level of billing met by a note, the templates can be used to comply with billing and require evaluation and management visits. As data are collected either prior to or during a patient encounter, the template can be completed.

How templates are created is an important issue with EMRs. Every physician has slightly different views as to what constitutes a perfect template. To meet the needs of different practitioners and practices, an EMR should allow the use of templates, which can be modified to meet the individual requirements of specific providers. EMRs directed to large group practices often impose a control on the modification of templates because of concern of too many variations. In smaller practices, individual modification is less a concern and should enhance program retention. Many programs allow for revising or modifying templates "on the run" to accommodate changes in procedures or techniques.

The creation of templates can be an expensive process. Ensure that the software developer has a template acceptable to your practice prior to buying the software.

\section{Software Developer or Value-Added Reseller}

A related issue is whether to buy from a manufacturer or from a value- added reseller (VAR). The manufacturer has a vested interested in a successful implementation of the program and in your success. Assuming that the manufacturer has ridden the learning curve before you, you should expect the dealer to provide a timely installation of a program that works and to support your hardware-software and network solution. VARs should, theoretically, be able to provide similar expert advice and implementation. The data exchange and management field is poorly regulated, and VARs should be thoroughly reviewed before the decision is made to go with them.

\section{Support}

Supporting transitioning to an EMR is a difficult process for both physician and staff. The process is not intuitive, and the presence of a definite learning curve affects how soon the practice can see enhanced productivity (29). Whether this curve is successfully ridden is a function of the support provided by a vendor. A significant portion of this support should be provided in the office. Seeing how an individual practice handles workflow that allows the vendor to enhance both the training process and the chances of retention has advantages. After the initial training period, handling remotely any of the inevitable problems that occur with a system, along with upgrades, should be possible over the Internet.

\section{Multiple Sites}

Depending on the practice, the EMR may be needed for use in multiple sites. One practitioner may work in several settings, or multiple practitioners may work in several settings, and databases generated at these different sites are replicated. A fundamental concern is latency: how long can the practice wait until the data are synchronized or replicated between different sites? If a practice has two practitioners who neither see each other's patients nor take calls for each other, the data may never need to be replicated at the level of the EMR. Merely transmitting their disparate billing information to the PMS software may be sufficient. Practitioners who go to a remote site on Friday may be satisfied by using a laptop at the remote site and then hooking the laptop into the main office network on Monday, replicating data at that time. If all practitioners in a practice need to link into one database, synchronization is possible using the In- ternet with firewall protection and a highspeed connection. With the widespread availability of DSL or cable connections, a T1 line is probably unnecessary, unless one is using Voice of Internet Protocol (VOIP) phones and is looking for the enhanced reliability of T1 lines over DSL.

\section{Document Management}

A neglected aspect of running a medical practice is document management. With paper charts, data are simply placed in the chart, often according to a very specific format favored by the practitioner. The issue of document management becomes problematic with EMRs. Initially, most practices are best advised to ignore the issue and retain the outside referral information and procedure notes in a paper folder, under the theory that you should be able to reduce your paper by $80 \%$ but not totally.

Eventually, one might wish to go to a paperless office. To have a completely paperless office, one needs digital document management, which allows certain advantages:

- Rapid retrieval of data, allowing a review of current medical status without a time lag

- Efficient importing of information into the chart, preferably by accepting it from a remote source in a digitalized format, such as a fax, so that office personnel do not need to rescan it. Many radiology departments are now storing images digitally.

- Easy location of data and, if necessary, easy exporting of the data

For the most part, current EMRs do not adequately handle document management. Adobe is too slow to allow the rapid review of extensive charts, and its filing system is rudimentary. Other document management programs geared to major enterprise users, such as national mortgage lenders or the admissions offices of university systems, do exist. Although excellent, they tend to operate on a scale larger than necessary for the standard medical practice, and they are expensive. EMR developers are aware of the document management problem. At least one vendor already has an integrated, proprietary document management system; others are collaborating with existing commercial providers to develop such systems for EMRs. Physicians should anticipate that with the next generation of software, 
we should have adequate documentation software to have a fully paperless office. Even though a paperless office will solve many problems, the downside of the paperless office is storage. Although storage is relatively cheap, it is still an expense. In like manner, also anticipate that your laptop may lack sufficient storage to handle the entirety of your medical charts.

The proper focus of a physician considering implementing information technology into medical office practice should be choosing and purchasing the electronic medical record practice management software and documentation management solutions.

\section{Practical Considerations for the Medical Practice}

The first step in transitioning to an EMR is to develop a consensus among the practice's decision makers as to the group's goals (30). Where do you plan to get efficiencies and how does the EMR fit in with your practice structure? Everyone wants lower costs but, realistically, how will this occur? A solo practice, for example, whose employees are family members is unlikely to realize major savings from reduction of payroll. Understand the structure of your practice and your workflow sufficiently to absorb what can be provided by an EMR. In addition, be prepared to spend a sizable sum of money on the software; anticipate that the costs of implementation are higher than the vendor would suggest; discount pro formas that illustrate discounted rates of return of several hundred percent. These proposed rates will be decreased by the increased costs generated by the time it takes you to "go ride the learning curve" or to investigate numerous systems. Accept that these capital expenditures will be spread out over time. Additionally, remember the tax benefit. Choosing the right system is difficult. All systems, at first glance, do all things. There is no easy way to discern which system will best suit your needs. At this point, with relatively few practices currently using EMRs, and the market growing, no one obvious solution exists to the problem of choosing a system to use in a pain management practice. If a physician chooses the top ten programs and reviews their websites, the programs blur into meaningless similarity. Unfortunately, understanding the subtleties of a program is very difficult until after you have purchased it and have be- gun working with it. Regrettably, no program at this point is perfect.

Adaptation of EMRs is limited, but we are beyond the pioneer stage. The best way to confirm that your choice is sound and that you have maximized your chances of success is to check with a practice that is similar to yours and has already implemented the program.

\section{FUTURE ISSUES}

EMRs are not yet perfect, as attested to by current low implementation and retention rates. They continue to improve rapidly. As market leaders emerge, the market will see convergence of the features offered by various systems and software developer consolidation.

Significant enhancement can be expected in two major areas: data input and outcomes management-decision support capabilities.

\section{Data Input}

This article has already addressed the present problems of entering data; this will continue to be a major concern. Voice recognition hints at being the Holy Grail, but it is certainly not at the current time. Inaccuracies in dictation because of shortcomings of voice recognition are unacceptable. We are unaware of any system of data entry that equals its potential. For years, promises have been made that easily usable, highly accurate voice recognition software for the masses was just around the corner. Unfortunately, these promises have not been realized. Hopefully, as the sophistication of the software, the speed and storage capabilities of the hardware, and the accuracy and discernment of the microphones and sound cards continue to improve, we should move closer to accurate input and output.

Even with accurate voice recognition, the need for alternate sources of data entry will continue, including those of point-and-click drop-down lists, typing, and scanning information.

\section{Outcomes Management/Decision Support Capability}

The practice of interventional pain management is an interdisciplinary, rapidly evolving field. Chronic pain, which by definition has a long course of treatment, is frustrating for patients and payors alike because of the difficulty in documenting improvement. A major barrier to documenting improvement is the diffi- culty in tracking a patient's progress. The risk we face is that insurers will look at long-term treatment, without document of efficacy, as "low-lying fruit," a ripe source of savings, and diminish the access of our patients to necessary care.

The EMR should be able to provide this outcome data and to develop and document the effectiveness, or lack of effectiveness, of treatments. Quality assurance markers, such as functional indices, medication usage patterns, patient satisfaction, and work status, are infrequently measured common markers of clinical success. Criteria used by the American Agency for Healthcare Research and Quality to evaluate outcomes do not reflect the evolving nature of most pain clinicians' practices (12). The current approach to outcomes management among many pain practitioners-"I think it, therefore it is"-yields little clinical significance when documentation and validation are required to ensure robust viability. The EMR provides hope that greater accountability through data acquisition and retrieval will aid in understanding pain treatment strategies, which direct clinical decision making.

The next step after outcomes management and analysis is decision support systems: the ability of the system to understand where the patient is in the treatment algorithm and to suggest, within the confines of evidence-based medicine, treatment alternatives. Added documentation of medical necessity and support of decision making enhances patient care and reimbursement potential.

\section{ConClusion}

Electronic medical records will be widely adopted into medical practices for reasons of safety, quality of care, compliance, productivity, and quality of life. This transition has support at the highest levels.

EMRs comprise both practice management software and the clinical record. The clinical EMR should, according to the Institute of Medicine, handle the patient's health information, the results of tests, and orders for medications, tests, and referrals; provide reminders of best practice care and such risk factors as drug interactions; be able to communicate securely among providers; and provide patient education and reporting sufficient to comply with various regulatory agencies. 
Currently, a multitude of competing providers are offering EMR software. Although the market can be expected to coalesce, with clear market leaders emerging, this consolidation has not yet occurred. Accordingly, the process of selecting EMRs is difficult and time consuming. EMRs are likely to be sold by vendors rather than purchased by providers. To avoid being sold a product that does not meet the needs of a practice, physicians should evaluate products on the basis of a clear understanding of their practice needs.

Among the specific issues to consider are whether to have the EMR and PMS functions handled by one program or two; to consider how well the software handles the practice's workflow; how data are entered into the program; what hardware and database solutions are offered; how templates are handled; whether to buy from the developer or from a VAR; how strong the developer's support program is; and what the needs are, if any, for multiple sites and how documents are managed.

EMRs are rapidly evolving and should have significant improvements in data input. Perhaps the greatest unrealized potential with EMRs is with outcomes management and decision support. These have the potential both of documenting the efficacy of procedures and therapies and of ensuring that the procedures or therapies recommended are consistent with evidence-based medicine.

The transition to EMRs will occur. With diligence, interventional pain management practices can make this transition, to the betterment of the practices and of patient care.

\section{ACKNOWLEDGEMENTS}

We acknowledge and thank Jeannette Keane for her editorial assistance in creating this document.

We also wish to thank editors for their review, criticism, and suggestions.

\section{Author Affiliation: \\ Standiford Helm II, MD}

Medical Director

Pacific Coast Pain Management Center

23792 Rockfield Blvd, Suite 101

Lake Forest, CA 92630

E-mail: drhelm@pcpmc.com

\section{Hans C. Hansen, MD}

Medical Director

The Pain Relief Centers, PA

3451 Graystone Place SE

Conover, NC 28613

E-mail hans@hippocrates.org

\section{REFERENCES}

1. Hassey AD, Gerrett A, Wilson A. A survey of validity and utility of electronic patient records in a general practice. $B M J$ 2001; 322: 1401-1405.

2. Wenner AR. North Carolina Academy of Family Physicians, Inc., Mid-Summer Family Medicine Digest: 20 Electronic Medical Record Pearls. Kingston Plantation, June 29-July 5, 2003:11-13.

3. Terry K. EMRs - What you need to know: There's a lot more to buying one than you might think. Know what you need before you go shopping. Med Econ 2003; 80: $\mathrm{TCP}_{5}-\mathrm{TCP}_{14}$.

4. Hippisley-Cox J, Pringle M, Cater R, et al. The electronic patient record in primary care-regression or progression? A cross sectional study. BMJ 2003; 326:14391343.

5. Hodges J. Medical records. Destination EMR. Hosp Health Netw 2003; 77:29-30.

6. Chin T. Small practices fuel sales of EMR systems. AMNews.com Feb. 9, 2004.

7. Jan 14, 2004. http://www.wellpoint.com/ $\mathrm{xml} / \mathrm{c} \_$newsreleasetext.asp?

8. George W. Bush. State of the Union Address, Jan. 20, 2004.

9. President launches push for electronic records; funds for project still in question. Bush wants most Americans to have an EMR within a decade. By Markian Hawryluk, AMNews staff. May 17, 2004.

http://www.ama-assn.org/amednews/ 2004/05/17/gvsa0517.htm

10. Hawryluk, M "Government pushes for electronic record standards: Legislation would help fund system purchases and ensure that users could share data." AMNews.com Feb. 9, 2004. http: //www.ama-assn.org/amednews/2004/ 02/09/gvsa0209.htm

11. Doscher M. HIPAA, A short- and longterm perspective for health care. Chicago: American Medical Association Press, 2002: p. 35.

12. http://www.h17.org/. HL7 (Health Level 7) is a standard developed by a nonprofit, volunteer organization. The mis- sion of $\mathrm{HL} 7$ is "To provide standards for the exchange, management and integration of data that support clinical patient care and the management, delivery and evaluation of healthcare services. Specifically, to create flexible, cost effective approaches, standards, guidelines, methodologies, and related services for interoperability between healthcare information systems."

13. Rose EA, Deshikachar AM, Schwartz KL, et al. Use of a template to improve documentation and billing. Fam Med 2001; 33:516521.

14. Manchikanti L (ed). Interventional pain medicine: documentation, billing, and coding - a practical guide for physicians and ASC's. Paducah, KY: ASIPP Publishing, 2002.

15. www.cms.hhs.gov/manuals/pm_trans/ R1764B3.pdf.

16. Kellum S, Gillmer B. Council corner. The benefits of an electronic patient record. Tar Heel Nurse 2000; 62:26-27.

17. Darbyshire P. User-friendliness of computerized information systems. Comput Nurs 2000; 18:93-99.

18. The Connected Physician: Technology Update-Special Technology Section. Chapters $4,10,12,15,17,18$.

19. Bates D, Gawande A. Patient safety: Improving safety with information technology. N Engl J Med 2003; 348:2526-2534.

20. http://www.bankrate.com/brm/itax/ Edit/tips/Stories/sec179_deduction.asp.

21. How We Evaluate Electronic Medical Records. Electronic Medical Records. 22 July $2003<$ <ww.elmr-electronic-medical-records-emr.com $>$.

22. Pallawala PM, Lun KC. EMR based tele-geriatric system. Int J Med Inform 2001; 61(23):229-234.

23. Mehta A, McLoud T. Voice recognition. J Thorac Imaging 2003; 18:178-182.

24. Ward L, Innes M. Electronic medical summaries in general practice-considering the patient's contribution. Br / Gen Pract 2003; 53:293-297.

25. Primer on Electronic Medical Records. Electronic Medical Records. 22 July 2003 www.elmr-electronic-medical-recordsemr.com.

26. Electronic Medical Record (EMR) Comparisons By Physicians for Physicians http: //www.elmr-electronic-medical-recordsemr.com/

27. Personal communication, May 20, 2004 Paul Riches, CA Board of Pharmacy Chief of Legislation and Regulation.

28. Product Descriptions. http://www.elmrelectronic-medical-records-emr.com// descriptions.htm. 22 July, 2003.

29. Microsoft corporation. <http:// www.microsoft.com/>.

30. Souther E. Implementation of the electronic medical record. The team approach. Comput Nurs 2001; 19:47-55. 